\title{
Development of the N1-P2 auditory evoked response to amplitude rise time and rate of formant transition of speech sounds
}

\author{
Allen L. Carpenter and Antoine J. Shahin ${ }^{*}$ \\ Department of Otolaryngology - Head \& Neck Surgery, The Ohio State University, Columbus, $\mathrm{OH}$ \\ 43212, United States
}

\begin{abstract}
We investigated the development of weighting strategies for acoustic cues by examining the morphology of the N1-P2 auditory evoked potential (AEP) to changes in amplitude rise time (ART) and rate of formant transition (RFT) of consonant-vowel (CV) pairsin4-6-year olds and adults. In the AEP session, individuals listened passively to the CVs /ba/, /wa/, and a /ba/ with a superimposed slower-rising /wa/ envelope $\left(/ \mathrm{ba} /{ }^{\mathrm{wa}}\right)$. In the behavioral session, individuals listened to the same stimuli and judged whether they heard a /ba/ or /wa/. We hypothesized that a developmental shift in weighting strategies should be reflected in a change in the morphology of the N1-P2 AEP. In 6-year olds and adults, the N1-P2 amplitude at the vertex reflected a change in RFT but not in ART. In contrast, in the 4-5-year olds, the vertex N1-P2 did not show specificity to changes in ART or RFT. In all groups, the N1-P2 amplitude at channel C4 (right hemisphere) reflected a change in ART but not in RFT. Behaviorally, 6-year olds and adults predominately utilized RFT cues (classified /ba/wa as /ba/) during phonetic judgments, as opposed to 4-5-year olds which utilized both cues equally. Our findings suggest that both ART and RFT are encoded in the auditory cortex, but an N1-P2 shift toward the vertex following age 4-5 indicates a shift toward an adult-like weighting strategy, such that, to utilize RFT to a greater extent.
\end{abstract}

\section{Keywords}

Auditory evoked potential; Consonant-vowel; Development; Speech; Amplitude rise time; Formant transition

\section{Introduction}

Spoken language processing entails encoding the many acoustic (temporal and spectral) cues afforded by the speech signal in auditory cortex (AC) and perceptually organizing them to enhance intelligibility. The current knowledge about how the relative weighting of acoustic cues changes with development is still a work in progress [3,7,11,12,14,17,20-22]. A study by Nittrouer et al. [12] supported the premise that children's reliance on the dynamics of formants develops before that of amplitude envelope in phonetic labeling. However, a recent study by Goswami et al. [7] showed that amplitude rise time (ART, how quickly acoustic

\section{(C) 2013 Elsevier Ireland Ltd. All rights reserved.}

"Corresponding author at: Department of Otolaryngology - Head \& Neck Surgery, The Ohio State University, 915 Olentangy River Road, Columbus, OH 43212, United States. Tel.: +1 614366 1019; fax: +1 614293 9667. tonyshahin@gmail.com (A.J. Shahin).

Conflict of interest statement: The authors declare that there is no conflict of interest.

Appendix A. Supplementary data: Supplementary data associated with this article can be found, in the online version, at http:// dx.doi.org/10.1016/j.neulet.2013.03.041. 
energy changes at speech onset) is crucial for development of language processing. They examined phonetic contrast abilities for synthetic/ba/and /wa/ consonant-vowels (CVs) that varied along their continuum of ART or rate of formant transition (RFT) in normal and dyslexic children. They reported that normal children were better at phonetic discrimination when the CVs varied in ART but not RFT [7], signifying the importance of amplitude information for phonetic labeling in normally developed children. We should note that similar studies $[13,24]$ on adults have shown that they predominately base their labeling of / ba/-/wa/ on RFT.

These previous, sometimes divergent, behavioral accounts motivated us to assess the neurophysiological causes of developmental shifts associated with weighting of ART and RFT. We measured the N1-P2 auditory evoked potential (AEP) to /ba/ and /wa/ CVs and a / $\mathrm{ba} / \mathrm{CV}$ superimposed with the slower-rising /wa/ envelope $\left(/ \mathrm{ba} /{ }^{\mathrm{wa}}\right)$ in children (4-6-year olds) and adults. The N1 and P2, which represent synchronous neural activity in the primary and non-primary AC [19], begin to emerge around 3-6 years of age [16,18,20]. Some studies have reported that this AEP complex emerges even later [21], while others place the milestone at a much younger age, $\sim 6$ months [4]. These differing accounts may be attributed partly to differences in experimental designs (rate of stimulus presentation, type of stimulus). Also, it is not clear whether the N1-P2 observed in infants [4] originates from the same neural generators that underlie the N1-P2 in older children. Notwithstanding, the magnitude and latency of the N1-P2 are known to reflect changes in spectrum and/or amplitude cues of CVs $[8,15]$. Thus, studying the N1-P2 developmental dynamics could help explain the maturation of children's weighting of acoustic cues during phonetic categorization.

We hypothesized that the N1-P2 morphology should reflect the weighting of ART and RFT. That is, when phonetic labeling is based heavily on RFT, the N1-P2 amplitude for /ba/wa should be similar to that of /ba/ (similar RFT, different ART) but different from that of /wa/ (similar ART, different RFT). However, if phonetic labeling is heavily based on ART, we should find that the N1-P2 amplitude of /ba/wa similar to that of /wa/ but different from that of $/ \mathrm{ba} /$. The first hypothesis is favored for adults. However, we expected children to use ART to a larger extent than adults, such that they may label /ba/wa as /ba/ or /wa/ equally. Hence, in children, the N1-P2 for /ba/wa may be different from those evoked by either /ba/ or /wa/.

\section{Materials and methods}

\subsection{Subjects}

Twenty-seven native English speakers were recruited from The Ohio State University via posted flyers and from a subject pool from the Department of Otolaryngology. Subjects were of the following ages: 4-6 years (between 4-years 0-months and 6-years and 11months; $n=15,8$ males) and 18-25 years ( $n=12,6$ males). Informed written consent was obtained from each participant, and parental consent was obtained for children. Normal audiometric thresholds for pure tones $(250-8000 \mathrm{~Hz},<25 \mathrm{~dB})$ were obtained for all participants. All experimental procedures were approved by the Institutional Research Board (IRB) of The Ohio State University.

\subsection{Stimuli}

Stimuli were recorded using Shure KSM microphone (Shure Inc., Niles, IL) and Tube MP Studio V3 pre-amplifier (ART, Rochester, NY), sampled at 48,000 Hz, and 16 bit from two native English speakers (female: $f_{0}=250 \mathrm{~Hz}$; male: $f_{0}=125 \mathrm{~Hz}$ ). All stimuli were $300 \mathrm{~ms}$ in duration, low-pass filtered at $12,000 \mathrm{~Hz}$, and of equal RMS power. The stimuli (see Supplemental Figure) consisted of 2 naturally voiced CVs, /ba/ and /wa/, and a natural /ba/ 
with a super-imposed /wa/ temporal envelope (termed/ba/wa). The/ba/ and/wa/ CVs exhibit similar formant trajectory (direction) but the rate of transition of the first and especially the second formant is delayed in $/ \mathrm{wa} /$. Thus, $/ \mathrm{ba} /$ and $/ \mathrm{ba} /{ }^{\text {wa }}$ differed in the temporal envelope but were similar in RFT, and /wa/ differed from /ba/wa only in RFT. The envelope superposition for /ba/wa was done using the Hilbert transform [1]. The behavioral and EEG testing also included additional CV types to test different hypotheses but are not discussed in this study.

\subsection{Procedure}

The experiment was optimized for the ERP passive task. Our goal was to show that development of perceptual encoding of acoustic cue weighting is reflected during low-level (bottom-up) auditory processing (in the absence of selective attention). This is hard to demonstrate using behavioral measures alone which require attention. Nonetheless, as a logical supplement, we also conducted a behavioral session to show that these low-level developmental changes transfer to behavior. Future developmental studies should take into account the top-down (selective attention) influence on development of AEP morphologies (e.g., passive vs. active tasks).

To control for order effects, half of the participants in each group began with the behavioral session and the other half began with the EEG session.

2.3.1. Behavior-We measured the classification accuracy of CVs for each subject. Subjects were seated in a sound-attenuated room and wore insert earphones (Etymotic Research ER4, Elk Grove Village, IL). First, each CV was presented separately, and the experimenter labeled the stimuli as /ba/ for /ba/, /wa/ for /wa/, and noise for noise. /ba/wa was labeled by the experimenter as a /ba/ "that may not be completely clear". The noise stimulus was included to control for guessing. That is, if a subject labeled a CV as noise more than $33 \%$ of the time, the participant was not included in the final analysis. Three 4year olds were excluded based on this criterion (six 4-5-year olds remained). Second, each $\mathrm{CV}$ type was presented randomly 8 times with instruction to identify whether the subjects heard all stimuli (/ba/, /wa/, /ba/wa or noise) as /ba/, /wa/, or noise. Subjects uttered their responses while the experimenter recorded them.

2.3.2. AEPs-Continuous electroencephalogram (EEG) was acquired using a 20-channel cap (10-20 system, BioSemi Active-2 system, Netherlands, sampled at $1024 \mathrm{~Hz}$, with Common Mode Sense and Driven Right Leg passive electrodes serving as grounds). Each $\mathrm{CV}$ was presented 50 times in a random order, in 4 blocks, for a total of 200 trials for each $\mathrm{CV}$ type (100 in a female voice and 100 in a male voice). A passive listening protocol was followed, during which subjects watched a subtitled silent movie of their choice on a monitor approximately one meter in front of them. Stimuli were presented at a comfortable sound-pressure level ( $\sim 60-65 \mathrm{~dB}$, according to initial feedback from participants) through the same earphones as the behavioral experiment. A variable inter-stimulus interval of 1.5-2 $\mathrm{s}$ was used. The EEG session lasted about 45 min with few minutes breaks between blocks.

\subsection{Data analysis}

Dramatic differences in behavior and AEPs prompted us to divide the children into two groups, 4-5-year olds (only one of which was 5-year old, $n=6$ ) and 6-year olds $(n=6)$.

2.4.1. Behavior-Correct classification for the natural /ba/, /wa/, and the $/ \mathrm{ba} /{ }^{\text {wa }}$ (labeled as /ba/) CVs were normalized to percentages of correct responses. 


\subsubsection{EEG}

2.4.2.1. Pre-processing: EEG analysis was conducted using EEGLAB [5] and in-house MATLAB (MathWorks, Inc., Natick, MA) code. Continuous EEG files were average referenced, epoched from $-200 \mathrm{~ms}$ to $+500 \mathrm{~ms}$ around stimulus onset, and baselined to the $200 \mathrm{~ms}$ pre-stimulus interval. Trials containing amplitudes of $\pm 150 \mu \mathrm{V}$ or larger at any channel were rejected. Subsequently, trials were averaged according to each CV type (collapsing over gender to increase number of trials and improve signal-to-noise ratio), and band-pass filtered between 0.5 and $30 \mathrm{~Hz}$ using a zero-phase FIR filter. Analysis of the N1 and P2 AEPs were performed at channels Fz, Cz, C3 and C4. The mean number and standard deviation of trials for each group (averaged across CV types) were $150 \pm 13$ for the 4-5-year olds; $184 \pm 11$ for the 6-year olds; $192 \pm 8$ for adults.

2.4.2.2. Peak analysis: Latencies of the N1 and P2 AEPs of the group average waveforms for each CV type were first identified visually at channels $\mathrm{Fz}, \mathrm{Cz}, \mathrm{C} 3$, and $\mathrm{C} 4$. The group latency for the N1 was identified as the maximum negative peak between $100 \mathrm{~ms}$ and 200 $\mathrm{ms}$ after sound onset for children and between $50 \mathrm{~ms}$ and $150 \mathrm{~ms}$ for adults. The group latency for the P2 was identified as the maximum positive peak between $140 \mathrm{~ms}$ and $250 \mathrm{~ms}$ for both children and adults. N1-P2 peaks could not be identified at C3 for the 4-5-year olds. Also, for the 4-5-year olds the group-average for two of the CVs did not show a clear $\mathrm{N} 1$ reversal (down and up potential), but they did show a deflection. In that case, the N1 and P2 amplitudes were determined as the beginning and end of the deflection, respectively. Subsequently, individual N1 and P2 peaks were automatically identified based on the group and $\mathrm{CV}$ type latencies identified above. The N1-P2 latencies at $\mathrm{C} 4$ were used to get individual N1-P2 peak values at C3 for the 4-5-year olds.

In younger children, the developing $\mathrm{N} 1$ is seen as an emerging small negative dip between the positive $\mathrm{P} 1$ and $\mathrm{P} 2$ peaks. Therefore, the $\mathrm{N} 1$ amplitude in young children is influenced by the amplitudes of the overlapping P1 and P2. To work around this problem the N1-P2 peak-to-peak amplitude, was considered as a more objective measure for comparing AEPs across age.

\subsection{Statistical analysis}

2.5.1. Behavior-An analysis of variance (ANOVA, general Linear Model of Statistic v. 9.1, StatSoft, OK) was conducted to show whether groups differed in their labeling of the $\mathrm{CV}$ s, with variables being group and $\mathrm{CV}$ type. The ANOVA tested the percentage that subjects labeled /ba/ as /ba/, /wa/ as /wa/, and /ba/wa as /ba/ correctly. Post hoc tests were based on Fisher's Least Significant Difference (LSD) test.

2.5.2. AEPs-N1-P2 peak-to-peak amplitudes for each channel were contrasted using an ANOVA with two factors: group and CV type. Post hoc analyses were based on LSD test.

\section{Results}

\subsection{Behavior}

Fig. 1A shows the percentages of correct CV classification for the three groups. An ANOVA on the correct responses revealed main effects of group $\left(F_{(2,21)}=59, p<0.0001\right.$; $\left.\eta_{\mathrm{p}}=0.85\right)$, and CV type $\left(F_{(2,42)}=10.5, p<0.0005 ; \eta_{\mathrm{p}}=0.33\right)$ and an interaction between the variables $\left(F_{(2,42)}=5.6, p<0.0005 ; \eta_{\mathrm{p}}=035\right)$. The group main effect was due to more correct labeling in the 6-year olds (>97\%) and adults (99\%) than the 4-5-year olds (71\%) (post hoc tests, $p<0.0001)$. The $\mathrm{CV}$ main effect was attributed to more correct responses for $/ \mathrm{ba} /$ and / wa/ $(>92 \%)$ than $/ \mathrm{ba} /{ }^{\mathrm{wa}}(79 \%)(p<0.005)$. There was no difference between the percentages of correct responses between $/ \mathrm{ba} /$ and $/ \mathrm{wa} /(p>0.5)$. Finally, the interaction between group 
and CV type was attributed to less accurate labeling of $/ \mathrm{ba} /{ }^{\text {wa }}$ (labeled as/ba/ $46 \%$; compare to correct labeling of/ba/ or /wa/ which was $>80 \%)$ in the $4-5$-year olds $(p<0.0001)$ but not in the older groups $(p>0.35$; Fig. 1A). In the 6 -year olds and adults, correct labeling of all 3 CVs was above $93 \%$. In short, the 6-year-olds and adults predominately labeled the CVs based on the RFT and ignored changes in ART, while the 4-5-year olds performed well when identifying the CVs with intact ART and RFT ( $~ 82 \%)$, but had trouble labeling /ba/wa (RFT as /ba/ and ART as /wa/).

\subsection{AEPs}

Fig. 1B shows AEPs at $\mathrm{Fz}, \mathrm{Cz}, \mathrm{C} 3$, and $\mathrm{C} 4$ averaged over all $\mathrm{CV}$ types for each group. N1 topographies are shown in Fig. 1B. Fig. 1C (left) shows the N1-P2 peak-to-peak amplitudes evoked by each CV type for each group at Cz. Fig. 1C (right) shows the N1-P2 amplitudes evoked by each $\mathrm{CV}$ type averaged across the three groups at channel $\mathrm{C} 4$. Notice from Fig. $1 \mathrm{~A}$ and $\mathrm{B}$, that for children, a positivity ("P1") dominates fronto-centrally and a negativity (N1) starts to emerge fronto-centrally (more at channel C4) at age 4-5, gets stronger (more bilaterally at $\mathrm{C} 3$ and $\mathrm{C} 4$ ) at age 6 , and fully dominates fronto-centrally for adults.

3.2.1. Fz-An ANOVA on the N1-P2 amplitudes at Fz revealed only a main effect of group $\left(F_{(2,21)}=19.5, p<0.0001 ; \eta_{\mathrm{p}}=0.6\right)$, and no interaction between the variables $(F<0.3)$. Post hoc analysis revealed that the group main effect was due to larger N1-P2 AEPs occurring in adults compared to children $(p<0.005)$ with no difference between the children's groups $(p>0.3)$.

3.2.2. $\mathrm{Cz}-\mathrm{An}$ ANOVA on the N1-P2 amplitudes at $\mathrm{Cz}$ revealed main effects of group $\left(F_{(2,21)}=17.1, p<0.0001 ; \eta_{\mathrm{p}}=0.62\right), \mathrm{CV}$ type $\left(F_{(2,42)}=6.0, p<0.005 ; \eta_{\mathrm{p}}=0.22\right)$, and an interaction between the variables $\left(F_{(4,42)}=5.5, p<0.005 ; \eta_{\mathrm{p}}=0.6\right)$. The group main effect was due to larger N1-P2s in adults compared to children $(p<0.005)$, with no difference between the children groups ( $p>0.2)$. The CV main effect was due to larger N1-P2s for /ba/ and $/ \mathrm{ba} /{ }^{\mathrm{wa}}$ compared to $/ \mathrm{wa} /(p<0.01)$, with no difference between AEPs for $/ \mathrm{ba} / \mathrm{and} / \mathrm{ba} /{ }^{\mathrm{wa}}$ $(p>0.2)$. Finally, the interaction between the variables was attributed to larger N1-P2s occurring for $/ \mathrm{ba} /$ and $/ \mathrm{ba} /{ }^{\text {wa }}$ compared to $/ \mathrm{wa} /$ only in the 6 -year olds and adults $(p<0.03)$. In these groups, the N1-P2s of/ba/ and /ba/wa did not differ ( $p>0.15)$. In short, the N1-P2s for the 6-year olds and adults were not different for CVs that had the same RFT and different ART, but differed for CVs of different RFTs. Finally, there was no difference between the emerging N1-P2 of the /ba/, /ba/wa and /wa/ CVs in 4-5-year olds ( $p>0.5$ ), suggesting a lack of specificity of the N1-P2 AEP at Cz to RFT or ART for this age group.

3.2.3. C3-N1-P2 at C3 exhibited similar behavior as Fz. Therefore, we do not state the statistics.

3.2.4. C4-An ANOVA on the N1-P2 amplitudes at $\mathrm{C} 4$ revealed main effects of group $\left(F_{(2,21)}=9.5, p<0.005 ; \eta_{\mathrm{p}}=0.47\right), \mathrm{CV}$ type $\left(F_{(2,42)}=6.5, p<0.005 ; \eta_{\mathrm{p}}=0.24\right)$, and no interaction between the variables $(F<0.5)$. The group main effect was due to larger N1-P2s occurring for adults compared to children $(p<0.01)$ with no difference between the children's groups ( $p>0.3)$. Interestingly, the CV main effect was attributed to larger N1-P2s occurring for the $/ \mathrm{ba} / \mathrm{compared}$ to $/ \mathrm{ba} /{ }^{\mathrm{wa}}$ and $/ \mathrm{wa} /(p<0.01)$ with no difference between the N1-P2s of the /ba/wa and /wa/ CVs ( $p>0.6)$. In short, the N1-P2 at C4 was sensitive to ART differences for all groups. This is opposite to the effect observed at $\mathrm{Cz}$ in the older groups. 


\section{Discussion}

Our results reveal that in children 6-years of age and adults the N1-P2 AEP at the vertex reflected the fidelity of RFT weighting. That is, the vertex N1-P2 was influenced by a change in the CVs' RFT but not ART. This was consistent with these groups' behavior, in which they almost exclusively relied on RFT, but not ART, in labeling the /ba/ /wa/ CVs. In contrast, there was no clear difference in the vertex N1-P2 AEP in the 4-5-year olds' in response to changes in RFT or ART, consistent with their chance level labeling of $/ \mathrm{ba} /{ }^{\mathrm{wa}}$. Interestingly, the N1-P2 at channel C4 (right hemisphere) reflected ART fidelity in all groups - the N1-P2 was influenced by a change in the CVs' ART but not RFT (opposite the effect seen at the vertex). In short, both ART and RFT are encoded in AC, but the emergence of stronger N1-P2 at the vertex points to a shift toward an adult-like weighting strategy, in which children begin to utilize RFT to a greater extent than ART.

This perceptual transition around age 4-6 years coincides with the manifestation of synaptic connections in the superficial layer of the AC [10], that are known to give rise to the N1-P2 complex. It also suggests that the N1-P2 begins to play an important role in encoding rapid spectral change (observed in $/ \mathrm{ba} /$ and $/ \mathrm{ba} /{ }^{\mathrm{wa}}$ ) within speech. Previous studies reported that theN1and/or P2 are sensitive to frequency change in the context of CV and vowel-vowel transition [6,8,9]. Martin et al. [9] showed that, in adults, the N1-P2 complex, evoked by a change of spectrum following vowel-to-vowel transition in /ui/, did not change during amplitude decrements. Their resultssuggestthattheN1-P2 evoked by formant transitions reflects cortical frequency encoding, consistent with our results for adults and 6-year olds (the vertex N1-P2 changed with RFT but not ART). Furthermore, the N1 and P2 response have been shown to augment following frequency [2] and voice onset time discrimination training [23], consistent with their roles in coding spectral complexity [20] and rapid spectral change. Thus, the emergence of the N1-P2 at the vertex following age 4-5 may indeed reflect the development of the cortical response associated with fine tuning to spectral cues in sound, which may cause children to develop reliance on spectral dynamics of speech (e.g., RFT).

An alternative explanation to our findings is that literacy-level (experience), as opposed to development, may have caused the children's shift in weighting strategy and AEPs. 4-5-year olds can be classified as pre-readers, while 6-year olds are being explicitly taught about letter-sound correspondences, which may have led to the development of phonemically specified representations. Such an explanation would fit the findings reported by Goswami et al. [7]. They reported that while children who struggle with reading (dyslexic) were poorer than age-matched controls at discriminating /ba/ from /wa/ on the basis of ART, they were not significantly different from younger reading-level matched controls. We should note however, that a comparison between our study and Goswami et al. [7] must be interpreted with caution because the two studies utilized different tasks, a discrimination task (same-different) vs. a labeling task. It is possible that children develop greater sensitivity to both ART and RFT with age (or experience), while at the same time develop a strategy that favors one cue over the other (RFT herein) during phonetic classification.

Our results do not suggest that a lack of a mature N1-P2 AEP is detrimental to speech comprehension. After all, 4-5-year olds understand natural speech well. However, conflicting acoustic cues could have an unfavorable impact on speech processing in children because of their weakly specified phonetic neural representations. Consequently, 4-5-year olds may need congruent acoustic cues in order to correctly label a phonetic sound. For example, when ART and RFT cues of a CV are not aligned with the stored cortical representations of that $\mathrm{CV}$, as in the $/ \mathrm{ba} /{ }^{\mathrm{wa}}$ stimulus, young children fail to integrate the two cues properly and their phonetic labeling suffers. Thus, our findings may suggest that the 
emergence of the vertex N1-P2 after age 4-5 allows a mechanism whereby children learn to segregate the cues and favor one over another.

There are two caveats of the current behavioral task: 1 - The 4-5-year olds may have been confused by the instructions. 2 - Number of trials per condition $(n=8)$, may have been inadequate. However, while we do advise prudence in future experiments, we are doubtful that these limitations confounded our conclusions, especially when taking into account a recent behavioral-only study that addressed the same question posed here [11]. Nittrouer et al. [11] examined labeling responses of /ba/-/wa/ along ART and RFT continua in 4-5-year olds and adults. When using the /ba/ stimulus with the slowest rising ART (most like /wa/ ART and most analogous to our $/ \mathrm{ba} /{ }^{\mathrm{wa}}$ ), $25 \%$ (compare to $50 \%$ in our study) of 4-5-year olds labeled it as /wa/, as opposed to adults which labeled it as $/ \mathrm{ba} / \sim 100 \%$ of the time. Despite this result, they [11] concluded that ART does not influence the /ba/-/wa/ contrast for children. Their conclusion however was based on all /ba/ conditions which included /ba/ CVs with ARTs that were less /wa/-like and more/ba/-like. Nonetheless, we argue that the two studies converge once taking into account the age difference between our 4 and 5 -year olds and theirs. In our study, five out of six of the 4-5-year olds were 4-years of age, as opposed to Nittrouer et al.'s [11], in which the age was more equally balanced between 4 and 5 years. If indeed ART is less weighted with age, as advocated in this study, then the greater number of 4-year olds in our 4-5-year old group, compared to Nittrouer's et al. [11], may have skewed the percentage to favor ART weighting in our study more than theirs.

\section{Conclusions}

Our findings provide evidence that after 4-5-years of age, children weight RFT more than ART (an adult-like strategy) during the /ba/-/wa/ phonetic categorization. This weighting shift toward greater reliance on spectral dynamics coincides with the emergence of the vertex N1-P2 AEP, reflecting synaptic maturation in the superficial layer of the AC. Accordingly, we posit that the period between 4 and 6 years of age represents a critical period in learning spoken language.

\section{Supplementary Material}

Refer to Web version on PubMed Central for supplementary material.

\section{Acknowledgments}

This study is supported by, and the design is documented in, an NIH/NIDCD grant (AJS, R03-DC011168). We thank Drs. Mark Pitt, Puisan Wong and Susan Nittrouer for helpful comments on the manuscript. We thank Jyoti Bhat and Dr. Joanna Lowenstein for their assistance. Results of this study were first reported (Carpenter, Nittrouer and Shahin) in an abstract at the Society for Neuroscience meeting in Washington DC November 2011.

\section{References}

1. Aiken SJ, Picton TW. Envelope and spectral frequency-following responses to vowel sounds. Hear Res. 2008; 245:35-47. [PubMed: 18765275]

2. Bosnyak DJ, Eaton RA, Roberts LE. Distributed auditory cortical representations are modified when non-musicians are trained at pitch discrimination with $40 \mathrm{~Hz}$ amplitude modulated tones. Cereb Cortex. 2004; 14:1088-1099. [PubMed: 15115745]

3. Ceponiene R, Rinne T, Naatanen R. Maturation of cortical sound processing as indexed by eventrelated potentials. Clin Neurophysiol. 2002; 113:870-882. [PubMed: 12048046]

4. Choudhury N, Benasich AA. Maturation of auditory evoked potentials from 6 to 48 months: prediction to 3 and 4 year language and cognitive abilities. Clin Neurophysiol. 2011; 122:320-338. [PubMed: 20685161] 
5. Delorme A, Makeig S. EEGLAB: an open source toolbox for analysis of single-trial EEG dynamics including independent component analysis. J Neurosci Methods. 2004; 134:9-21. [PubMed: 15102499]

6. Doellinger M, Burger M, Hoppe U, Bosco E, Eysholdt U. Effects of consonant-vowel transitions in speech stimuli on cortical auditory evoked potentials in adults. Open Neurol J. 2011; 5:37-45. [PubMed: 21643536]

7. Goswami U, Fosker T, Huss M, Mead N, Szucs D. Rise time and formant transition duration in the discrimination of speech sounds: the Ba-Wa distinction in developmental dyslexia. Dev Sci. 2011; 14:34-43. [PubMed: 21159086]

8. Korczak PA, Stapells DR. Effects of various articulatory features of speech on cortical event-related potentials and behavioral measures of speech-sound processing. Ear Hear. 2010; 31:491-504. [PubMed: 20453651]

9. Martin BA, Boothroyd A. Cortical, auditory, evoked potentials in response to changes of spectrum and amplitude. J Acoust Soc Am. 2000; 107:2155-2161. [PubMed: 10790041]

10. Moore JK, Guan YL. Cytoarchitectural and axonal maturation in human auditory cortex. J Assoc Res Otolaryngol. 2001; 2:297-311. [PubMed: 11833605]

11. Nittrouer, S.; Lowenstein, JH.; Tarr, E. Amplitude rise time does not cue the /ba/-/wa/ contrast for adults or children. J Speech Lang Hear Res. Published online September 19, 2012. http:// dx.doi.org/10.1044/1092-4388(2012/12-0075)

12. Nittrouer S, Lowenstein JH, Packer RR. Children discover the spectral skeletons in their native language before the amplitude envelopes. J Exp Psychol Hum Percept Perform. 2009; 35:12451253. [PubMed: 19653762]

13. Nittrouer S, Studdert-Kennedy M. The stop-glide distinction: acoustic analysis and perceptual effect of variation in syllable amplitude envelope for initial /b/ and /w. J Acoust Soc Am. 1986; 80:1026-1029. [PubMed: 3771922]

14. Ohde RN, Haley KL. Stop-consonant and vowel perception in 3- and 4-year-old children. J Acoust Soc Am. 1997; 102:3711-3722. [PubMed: 9407663]

15. Ostroff JM, Martin BA, Boothroyd A. Cortical evoked response to acoustic change within a syllable. Ear Hear. 1998; 19:290-297. [PubMed: 9728724]

16. Pang EW, Taylor MJ. Tracking the development of the N1 from age 3 to adulthood: an examination of speech and non-speech stimuli. Clin Neurophysiol. 2000; 111:388-397. [PubMed: 10699397]

17. Parnell MM, Amerman JD. Maturational influences on perception of coarticulatory effects. J Speech Hear Res. 1978; 21:682-701. [PubMed: 745369]

18. Ponton CW, Eggermont JJ, Kwong B, Don M. Maturation of human central auditory system activity: evidence from multi-channel evoked potentials. Clin Neurophysiol. 2000; 111:220-236. [PubMed: 10680557]

19. Scherg M, Von Cramon D. Two bilateral sources of the late AEP as identified by a spatio-temporal dipole model. Electroencephalogr Clin Neurophysiol. 1985; 62:32-44. [PubMed: 2578376]

20. Shahin AJ, Trainor LJ, Roberts LE, Backer KC, Miller LM. Development of auditory phase-locked activity for music sounds. J Neurophysiol. 2010; 103:218-229. [PubMed: 19864443]

21. Sussman E, Steinschneider M, Gumenyuk V, Grushko J, Lawson K. The maturation of human evoked brain potentials to sounds presented at different stimulus rates. Hear Res. 2008; 236:61-79. [PubMed: 18207681]

22. Sussman JE. Vowel perception by adults and children with normal language and specific language impairment: based on steady states or transitions? J Acoust Soc Am. 2001; 109:1173-1180. [PubMed: 11303931]

23. Tremblay K, Kraus N, McGee T, Ponton C, Otis B. Central auditory plasticity: changes in the N1P2 complex after speech-sound training. Ear Hear. 2001; 22:79-90. [PubMed: 11324846]

24. Walsh MA, Diehl RL. Formant transition duration and amplitude rise time as cues to the stop/glide distinction. Q J Exp Psychol A. 1991; 43:603-620. [PubMed: 1775659] 


\section{Highlights}

- 4-5-Year olds assign equal weights for formant and amplitude information.

- 6-year olds weight formant more than amplitude transition, an adult like strategy.

- A developmental shift in phonetic processing takes place around 4-6 years of age.

- The N1-P2 auditory response at the vertex reflects formant transition weighting.

- 4-6 years of age reflects a critical period in learning spoken language. 
A

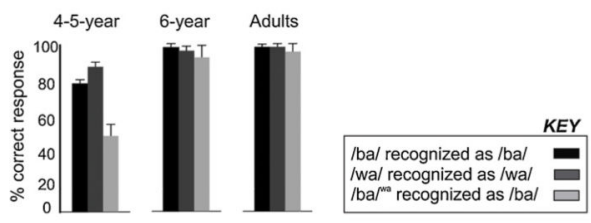

B

AEP waveforms

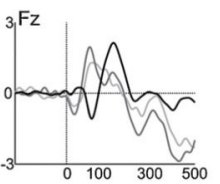

4-5-year olds -

6-year olds -
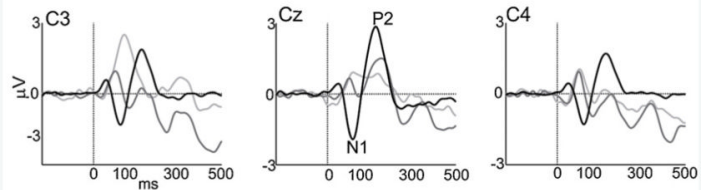

C

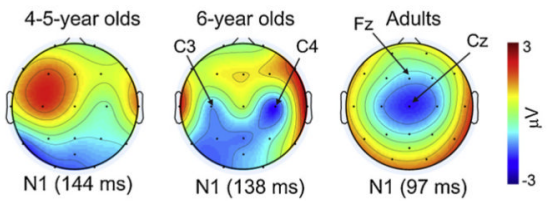

D

N1-P2 peak-to-peak amplitude

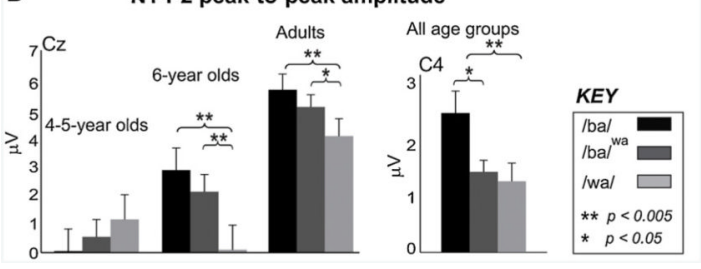

Fig. 1.

(A) Behavior: Percentages of correct classification of the CVs /ba/, /wa/ and /ba/wa , in 4-5year olds, 6 year olds and adults. Bars represent 1 standard error. (B) AEPs: Group averaged waveforms at channels $\mathrm{Fz}, \mathrm{Cz}, \mathrm{C} 3$, and $\mathrm{C} 4$ for the 4-5 and 6-year olds and adults, depicting auditory evoked potentials (AEPs) of all CVs (averaged over /ba/, /wa/ and /ba/wa). Vertical dashed lines at $0 \mathrm{~ms}$ represent sound onset. (C) Scalp topographies of the N1 wave for all groups. (D, left) The groups' N1-P2 peak-to-peak amplitudes evoked by the CVs /ba/, /wa/, and /ba/wa at channel Cz. (Right) The N1-P2 peak-to-peak amplitudes at channel C4 evoked by the three CVs averaged across the three groups. 